\title{
Fixed Point Theorems for an Elastic Nonlinear Mapping in Banach Spaces
}

\author{
Hiroko Manaka \\ Department of Mathematics, Graduate School of Environment and Information Sciences, Yokohama National University, \\ Tokiwadai, Hodogayaku, Yokohama 240-8501, Japan
}

Correspondence should be addressed to Hiroko Manaka; h-manaka@ynu.ac.jp

Received 17 October 2014; Accepted 8 December 2014

Academic Editor: Kyung Soo Kim

Copyright () 2015 Hiroko Manaka. This is an open access article distributed under the Creative Commons Attribution License, which permits unrestricted use, distribution, and reproduction in any medium, provided the original work is properly cited.

Let $E$ be a smooth Banach space with a norm $\|\cdot\|$. Let $V(x, y)=\|x\|^{2}+\|y\|^{2}-2\langle x, J y\rangle$ for any $x, y \in E$, where $\langle\cdot, \cdot\rangle$ stands for the duality pair and $J$ is the normalized duality mapping. We define a $V$-strongly nonexpansive mapping by $V(\cdot, \cdot)$. This nonlinear mapping is nonexpansive in a Hilbert space. However, we show that there exists a $V$-strongly nonexpansive mapping with fixed points which is not nonexpansive in a Banach space. In this paper, we show a weak convergence theorem and strong convergence theorems for fixed points of this elastic nonlinear mapping and give the existence theorem.

\section{Introduction}

Let $E$ be a smooth Banach space with a norm $\|\cdot\|$ and let $E^{*}$ be the dual space of $E$. We denote by $\langle\cdot, \cdot\rangle$ a duality pair on $E \times E^{*}$ and let $J$ be the normalized duality mapping on $E$. It is well known that $J$ is a continuous single-valued mapping in a smooth Banach space and a one-to-one mapping in a strictly convex Banach space (cf. [1]). We define a mapping $V: E \times$ $E \rightarrow \mathbb{R}$ by $V(x, y)=\|x\|^{2}+\|y\|^{2}-2\langle x, J y\rangle$ for all $x, y \in E$, where $\mathbb{R}$ is a set of real numbers. It is obvious that $V(x, y) \geq$ $(\|x\|-\|y\|)^{2} \geq 0$. Let any $y \in E$ be fixed, and then $V(\cdot, y)$ is a convex function because of convexity of $\|\cdot\|^{2}$. Many nonlinear mappings which are defined by using $V(\cdot, \cdot)$ are studied (see $[2-4])$. We also defined a nonlinear mapping which is called a $V$-strongly nonexpansive mapping in [5] as follows.

Definition 1. Let $C$ be a nonempty subset of a smooth Banach space $E$. A mapping $T: C \rightarrow E$ is called $V$-strongly nonexpansive if there exists a constant $\lambda>0$ such that for all $x, y \in$ C

$$
V(T x, T y) \leq V(x, y)-\lambda V((I-T) x,(I-T) y),
$$

where $I$ is the identity mapping on $E$.

From this definition, it is obvious that the identity mapping $I$ is also a $V$-strongly nonexpansive mapping. In a
Hilbert space, it is trivial that this mapping is nonexpansive since $V(x, y)=\|x-y\|^{2}$ and that any firmly nonexpansive mapping is a $V$-strongly nonexpansive mapping with $\lambda=1$ (see [5]). Moreover, we showed that if there exists a fixed point of a $V$-strongly nonexpansive mapping $T$, then $T$ is strongly nonexpansive with a Bregman distance in [5]. However, in Banach spaces, as we give an example in the later section, we find that there exists a $V$-strongly nonexpansive mapping with fixed points which is not nonexpansive. We should point out that a guarantee of continuity of the $V$-strongly nonexpansive mappings has not been given in a generalized Banach space yet.

In this paper, we prove a weak convergence theorem and strong convergence theorems for finding fixed points of a $V$-strongly nonexpansive mapping in Banach spaces and show the existence theorem of fixed point with a dissipative property.

\section{Preliminaries}

In this section, at first we show the relationship between a $V$-strongly nonexpansive mapping and other nonlinear mappings, in a Hilbert space. Secondly, we state some properties of $V$-strongly nonexpansive mappings in a Banach space and give an example of a $V$-strongly nonexpansive mapping 
which is not a quasinonexpansive mapping in a Banach space although $T$ has fixed points. We finally show some lemmas which are necessary in order to prove our theorems.

Let $C$ be a subset of a Banach space $E$ and let $T: C \rightarrow E$ be a mapping. Then a point $p$ in the closure of $C$ is said to be an asymptotically fixed point of $T$ if $C$ contains a sequence $\left\{x_{n}\right\}$ which converges weakly to $p$ and the sequence $\left\{x_{n}-T x_{n}\right\}$ converges strongly to $0 . \widehat{F}(T)$ denotes the set of asymptotically fixed points of $T$. In [6], Reich introduced a strongly nonexpansive mapping which is defined by using the Bregman distance $D(\cdot, \cdot)$.

Definition 2. Let $E$ be a Banach space. The Bregman distance corresponding to a function $f: E \rightarrow \mathbb{R}$ is defined by

$$
D(x, y)=f(x)-f(y)-f^{\prime}(y)(x-y),
$$

where $f$ is Gâteaux differentiable and $f^{\prime}(x)$ stands for the derivative of $f$ at the point $x$. Let $C$ be a nonempty subset of $E$. We say that the mapping $T: C \rightarrow E$ is strongly nonexpansive if $\widehat{F}(T) \neq \emptyset$ and

$$
D(p, T x) \leq D(p, x) \quad \forall p \in \widehat{F}(T) \quad x \in C,
$$

and if it holds that $\lim _{n \rightarrow \infty} D\left(T x_{n}, x_{n}\right)=0$ for a bounded sequence $\left\{x_{n}\right\}$ such that $\lim _{n \rightarrow \infty}\left(D\left(p, x_{n}\right)-D\left(p, T x_{n}\right)\right)=0$ for any $p \in \widehat{F}(T)$.

Taking the function $\|\cdot\|^{2}$ as the convex, continuous, and Gâteaux differentiable function $f$, we obtain the fact that the Bregman distance $D(\cdot, \cdot)$ coincides with $V(\cdot, \cdot)$. In particular, in a Hilbert space, it is trivial that $D(x, y)=V(x, y)=\| x-$ $y \|^{2}$.

Proposition 3 (see [5]). In a Hilbert space, a $V$-strongly nonexpansive mapping with $\widehat{F}(T) \neq \emptyset$ is strongly nonexpansive.

Next we recall two mappings of other nonlinear mappings (cf. [6-9]). A firmly nonexpansive mapping and an $\alpha$-inverse strongly monotone mapping are defined as follows.

Definition 4. Let $C$ be a nonempty, closed, and convex subset of a Banach space $E$. A mapping $T: C \rightarrow E$ is said to be firmly nonexpansive if

$$
\|T x-T y\|^{2} \leq\langle x-y, j\rangle
$$

for all $x, y \in C$ and some $j \in J(T x-T y)$.

It is trivial that a firmly nonexpansive mapping is nonexpansive.

Definition 5. Let $H$ be a Hilbert space. A mapping $T: C \rightarrow$ $H$ is said to be $\alpha$-inverse strongly monotone if

$$
\alpha\|T x-T y\|^{2} \leq\langle x-y, T x-T y\rangle
$$

for all $x, y \in C$.

The relation among firmly nonexpansive mappings, $\alpha$ inverse strongly monotone mappings and $V$-strongly nonexpansive mappings is shown in the following proposition.
Proposition 6 (see [5]). In a Hilbert space, the following hold.

(a) A firmly nonexpansive mapping is $V$-strongly nonexpansive with $\lambda=1$.

(b) Let $A$ be an $\alpha$-inverse strongly monotone mapping for $\alpha>1 / 2$; then $S=(I-A)$ is $V$-strongly nonexpansive with $(2 \alpha-1)$.

The above (b) is obvious by showing that, for all $x, y \in H$,

$$
\langle S x-S y, x-y\rangle \leq\|x-y\|^{2}-\alpha\|(I-S) x-(I-S) y\|^{2} .
$$

We will introduce some properties of $V$-strongly nonexpansive mappings in [5].

Proposition 7 (see [5]). In a smooth Banach space E, the following hold.

(a) For $c \in(-1,1], T=c I$ is $V$-strongly nonexpansive. For $c=1, T=I$ is $V$-strongly nonexpansive for any $\lambda>0$. For $c \in(-1,1), T=c I$ is $V$-strongly nonexpansive for any $\lambda \in(0,(1+c) /(1-c)]$.

(b) If $T$ is $V$-strongly nonexpansive with $\lambda$, then, for any $\alpha \in[-1,1]$ with $\alpha \neq 0, \alpha T$ is also $V$-strongly nonexpansive with $\alpha^{2} \lambda$.

(c) If $T$ is $V$-strongly nonexpansive with $\lambda \geq 1$, then $A=$ $I-T$ is $V$-strongly nonexpansive with $\lambda^{-1}$.

(d) Suppose that $T$ is $V$-strongly nonexpansive with $\lambda$ and that $\alpha \in[-1,1]$ satisfies $\alpha^{2} \lambda \geq 1$. Then $(I-\alpha T)$ is $V$ strongly nonexpansive with $\left(\alpha^{2} \lambda\right)^{-1}$. Moreover, if $T_{\alpha}=$ $I-\alpha T$, then

$$
V\left(T_{\alpha} x, T_{\alpha} y\right) \leq V(x, y)-\lambda^{-1} V(T x, T y) .
$$

Now we give an example of a $V$-strongly nonexpansive mapping in a Banach space.

Example 8 (see [10]). Let $1<p, q<\infty$ such that $1 / p+1 / q=$ 1 . Let $E=\mathbb{R} \times \mathbb{R}$ be a real Banach space with a norm $\|\cdot\|_{p}$ defined by

$$
\|x\|_{p}=\left\{\left|x_{1}\right|^{p}+\left|x_{2}\right|^{p}\right\}^{1 / p} \quad \forall x=\left(x_{1}, x_{2}\right) \in E .
$$

Then $E$ is smooth, and the normalized duality mapping $J$ is single-valued. $J$ is given by

$$
\begin{array}{r}
J x=\|x\|_{p}^{2-p}\left(x_{1}\left|x_{1}\right|^{p-2}, x_{2}\left|x_{2}\right|^{p-2}\right) \in l^{q}(\mathbb{R} \times \mathbb{R}) \\
\forall x=\left(x_{1}, x_{2}\right) \in E .
\end{array}
$$

Hence, we have for $x, y \in E$ that

$$
\begin{aligned}
V(x, y)= & \|x\|_{p}^{2}+\|y\|_{p}^{2}-2\langle x, J y\rangle \\
= & \|x\|_{p}^{2}+\|y\|_{p}^{2}-2\|y\|_{p}^{2-p} \\
& \cdot\left\{x_{1} y_{1}\left|y_{1}\right|^{p-2}+x_{2} y_{2}\left|y_{2}\right|^{p-2}\right\} .
\end{aligned}
$$


We define a mapping $T: E \rightarrow E$ as follows:

$$
T x= \begin{cases}x & \text { if }\|x\|_{p} \leq 1 \\ \frac{1}{\|x\|_{p}} x & \text { if }\|x\|_{p}>1 .\end{cases}
$$

In a case of $p=1$, we have shown that the mapping $T$ defined by (11) is a $V$-strongly nonexpansive mapping (see [5]). We will show that $T$ is $V$-strongly nonexpansive with any $\lambda \leq 1$, for $p>1$.

Proposition 9. Suppose that $T$ is defined by the formula (11) under the above situation. Then, $T$ is a $V$-strongly nonexpansive mapping with any $\lambda \leq 1$.

Proof. Case (a): suppose that $x, y \in E$ with $\|x\|_{p} \leq 1$ and $\|y\|_{p}>1$. that

Since $T y=\left((T y)_{1},(T y)_{2}\right)=\left(y_{1}\|y\|_{p}^{-1}, y_{2}\|y\|_{p}^{-1}\right)$, we have

$$
\begin{aligned}
V(T x, T y)= & V(x, T y)=\|x\|_{p}^{2}+\|T y\|_{p}^{2}-2\|T y\|_{p}^{2-p} \\
& \cdot\left\{x_{1}(T y)_{1}\left|(T y)_{1}\right|^{p-2}+x_{2}(T y)_{2}\left|(T y)_{2}\right|^{p-2}\right\} \\
= & \|x\|_{p}^{2}+1-2\|y\|_{p}^{1-p} \\
& \cdot\left\{x_{1} y_{1}\left|y_{1}\right|^{p-2}+x_{2} y_{2}\left|y_{2}\right|^{p-2}\right\}
\end{aligned}
$$

Since

$$
y-T y=\left(\frac{\|y\|_{p}-1}{\|y\|_{p}} y_{1}, \frac{\|y\|_{p}-1}{\|y\|_{p}} y_{2}\right),
$$

we have that

$$
\begin{aligned}
V(x-T x, y-T y) & =V(0, y-T y)=\|y-T y\|_{p}^{2} \\
& =\left\{\frac{\left(\|y\|_{p}-1\right)}{\|y\|_{p}}\|y\|_{p}\right\}^{2} \\
& =\left(\|y\|_{p}-1\right)^{2} .
\end{aligned}
$$

Hence, we obtain that

$$
\begin{aligned}
& V(x, y)-V(T x, T y)-\lambda V(x-T x, y-T y) \\
&=\|x\|_{p}^{2}+\|y\|_{p}^{2}-2\|y\|_{p}^{2-p}\left\{x_{1} y_{1}\left|y_{1}\right|^{p-2}+x_{2} y_{2}\left|y_{2}\right|^{p-2}\right\} \\
&-\|x\|_{p}^{2}-1+2\|y\|_{p}^{1-p}\left\{x_{1} y_{1}\left|y_{1}\right|^{p-2}+x_{2} y_{2}\left|y_{2}\right|^{p-2}\right\} \\
&-\lambda\left(\|y\|_{p}-1\right)^{2} \\
&=\|y\|_{p}^{2}-1-2\|y\|_{p}^{1-p}\left(\|y\|_{p}-1\right) \\
& \cdot\left\{x_{1} y_{1}\left|y_{1}\right|^{p-2}+x_{2} y_{2}\left|y_{2}\right|^{p-2}\right\}-\lambda\left(\|y\|_{p}-1\right)^{2}
\end{aligned}
$$

$$
\begin{aligned}
\geq\left(\|y\|_{p}-1\right)\{ & \left(\|y\|_{p}+1\right)-2\|y\|_{p}^{1-p} \\
& \cdot\left(\left|x_{1}\right|\left|y_{1}\right|^{p-1}+\left|x_{2}\right|\left|y_{2}\right|^{p-1}\right) \\
& \left.-\lambda\left(\|y\|_{p}-1\right)\right\} .
\end{aligned}
$$

Hölder's inequality implies that

$$
\begin{aligned}
\left|x_{1}\right|\left|y_{1}\right|^{p-1}+\left|x_{2}\right|\left|y_{2}\right|^{p-1} & \leq\|x\|_{p}\left\{\left(\left|y_{1}\right|^{p-1}\right)^{q}+\left(\left|y_{2}\right|^{p-1}\right)^{q}\right\}^{1 / q} \\
& =\|x\|_{p}\left(\left|y_{1}\right|^{p}+\left|y_{2}\right|^{p}\right)^{1 / q} \\
& =\|x\|_{p}\|y\|_{p}^{p-1} .
\end{aligned}
$$

Therefore, we obtain that

$$
\begin{aligned}
& V(x, y)-V(T x, T y)-\lambda V(x-T x, y-T y) \\
& \geq\left(\|y\|_{p}-1\right) \\
& \cdot\left\{\|y\|_{p}+1-2\|y\|_{p}^{1-p}\|x\|_{p}\|y\|_{p}^{p-1}-\lambda\|y\|_{p}+\lambda\right\} \\
&=\left(\|y\|_{p}-1\right)\left\{\|y\|_{p}+1-2\|x\|_{p}-\lambda\|y\|_{p}+\lambda\right\} \\
& \geq\left(\|y\|_{p}-1\right)\left\{(1-\lambda)\|y\|_{p}+1-2+\lambda\right\} \\
&=\left(\|y\|_{p}-1\right)\left\{(1-\lambda)\left(\|y\|_{p}-1\right)\right\} \\
&=(1-\lambda)\left(\|y\|_{p}-1\right)^{2} \geq 0, \quad \text { for any } \lambda \in[0,1] .
\end{aligned}
$$

That is, the inequality (1) holds.

Case (b): suppose that $x, y \in E$ with $\|x\|_{p} \geq 1$ and $\|y\|_{p} \leq$ 1.

Then we have that

$$
\begin{aligned}
V(T x, T y)= & V(T x, y) \\
= & 1+\|y\|_{p}^{2}-2\|x\|_{p}^{-1}\|y\|_{p}^{2-p} \\
& \cdot\left\{x_{1} y_{1}\left|y_{1}\right|^{p-2}+x_{2} y_{2}\left|y_{2}\right|^{p-2}\right\}, \\
V(x-T x, y-T y)= & V\left(\frac{\left(\|x\|_{p}-1\right)}{\|x\|_{p}} x, 0\right)=\left(\|x\|_{p}-1\right)^{2} .
\end{aligned}
$$

Hence, we have that

$$
\begin{aligned}
& V(x, y)-V(T x, T y)-\lambda V(x-T x, y-T y) \\
&=\|x\|_{p}^{2}+\|y\|_{p}^{2}-2\|y\|_{p}^{2-p} \\
& \cdot\left\{x_{1} y_{1}\left|y_{1}\right|^{p-2}+x_{2} y_{2}\left|y_{2}\right|^{p-2}\right\}-1-\|y\|_{p}^{2} \\
&+2\|y\|_{p}^{2-p}\|x\|_{p}^{-1}\left\{x_{1} y_{1}\left|y_{1}\right|^{p-2}+x_{2} y_{2}\left|y_{2}\right|^{p-2}\right\} \\
&-\lambda\left(\|x\|_{p}-1\right)^{2}
\end{aligned}
$$




$$
\begin{aligned}
\geq & \|x\|_{p}^{2}-1-2\|y\|_{p}^{2-p} \\
& \cdot\left\{\left|x_{1}\right|\left|y_{1}\right|^{p-1}+\left|x_{2}\right|\left|y_{2}\right|^{p-1}\right\}\left(1-\|x\|_{p}^{-1}\right) \\
& -\lambda\left(1-\|x\|_{p}\right)^{2} .
\end{aligned}
$$

As (a), we obtain from Hölder's inequality that

$$
\begin{aligned}
& V(x, y)-V(T x, T y)-\lambda V(x-T x, y-T y) \\
& \geq\|x\|_{p}^{2}-1-2\|x\|_{p}\|y\|_{p}^{2-p}\|y\|_{p}^{p-1} \\
& \cdot\left(1-\|x\|_{p}^{-1}\right)-\lambda\left(\|x\|_{p}-1\right)^{2} \\
&=\left(\|x\|_{p}-1\right)\left(\|x\|_{p}+1\right)-2\|y\|_{p}\left(\|x\|_{p}-1\right) \\
&-\lambda\left(\|x\|_{p}-1\right)^{2} \\
&=\left(\|x\|_{p}-1\right)\left\{\|x\|_{p}+1-2\|y\|_{p}-\lambda\|x\|_{p}+\lambda\right\} \\
& \geq\left(\|x\|_{p}-1\right)(1-\lambda)\left(\|x\|_{p}-1\right) \\
&=(1-\lambda)\left(\|x\|_{p}-1\right)^{2} \geq 0, \quad \text { for any } \lambda \in[0,1] .
\end{aligned}
$$

That is, the inequality (1) holds.

Case (c): suppose that $x, y \in E$ with $\|x\|_{p},\|y\|_{p} \geq 1$.

Then we have that

$$
\begin{aligned}
V(T x, T y) & \\
= & 1+1-2\left\langle\|x\|_{p}^{-1}\left(x_{1}, x_{2}\right),\right. \\
& \left.\|y\|_{p}^{1-p}\left(y_{1}\left|y_{1}\right|^{p-2}, y_{2}\left|y_{2}\right|^{p-2}\right)\right\rangle \\
= & 2-2\|x\|_{p}^{-1}\|y\|_{p}^{1-p}\left\{x_{1} y_{1}\left|y_{1}\right|^{p-2}+x_{2} y_{2}\left|y_{2}\right|^{p-2}\right\}, \\
V(x-T x, y-T y) & \\
= & V\left(\frac{\|x\|_{p}-1}{\|x\|_{p}} x, \frac{\|y\|_{p}-1}{\|y\|_{p}} y\right) \\
= & \left(\|x\|_{p}-1\right)^{2}+\left(\|y\|_{p}-1\right)^{2} \\
& -2\left(\|x\|_{p}-1\right)\left(\|y\|_{p}-1\right)\|x\|_{p}^{-1}\|y\|_{p}^{-1}\|y\|_{p}^{2-p} \\
& \cdot\left\langle\left(x_{1}, x_{2}\right),\left(\left|y_{1}\right|^{p-2} y_{1},\left|y_{2}\right|^{p-2} y_{2}\right)\right\rangle \\
= & \left(\|x\|_{p}-1\right)^{2}+\left(\|y\|_{p}-1\right)^{2} \\
& -2\left(\|x\|_{p}-1\right)\left(\|y\|_{p}-1\right)\|x\|_{p}^{-1}\|y\|_{p}^{1-p} \\
& \cdot\left\{x_{1} y_{1}\left|y_{1}\right|^{p-2}+x_{2} y_{2}\left|y_{2}\right|^{p-2}\right\} .
\end{aligned}
$$

Hence, we have that

$$
\begin{aligned}
& V(x, y)-V(T x, T y)-\lambda V(x-T x, y-T y) \\
&=\|x\|_{p}^{2}+\|y\|_{p}^{2}-2\|y\|_{p}^{2-p}\left\{x_{1} y_{1}\left|y_{1}\right|^{p-2}+x_{2} y_{2}\left|y_{2}\right|^{p-2}\right\} \\
&-2+2\|x\|_{p}^{-1}\|y\|_{p}^{1-p}\left\{x_{1} y_{1}\left|y_{1}\right|^{p-2}+x_{2} y_{2}\left|y_{2}\right|^{p-2}\right\} \\
&-\lambda\left(\|x\|_{p}-1\right)^{2}-\lambda\left(\|y\|_{p}-1\right)^{2} \\
&+2 \lambda\left(\|x\|_{p}-1\right)\left(\|y\|_{p}-1\right)\|x\|_{p}^{-1}\|y\|_{p}^{1-p} \\
& \cdot\left\{x_{1} y_{1}\left|y_{1}\right|^{p-2}+x_{2} y_{2}\left|y_{2}\right|^{p-2}\right\} \\
&=\|x\|_{p}^{2}+\|y\|_{p}^{2}-2-\lambda\left(\|x\|_{p}-1\right)^{2}-\lambda\left(\|y\|_{p}-1\right)^{2} \\
&-2\|x\|_{p}^{-1}\|y\|_{p}^{1-p}\left\{x_{1} y_{1}\left|y_{1}\right|^{p-2}+x_{2} y_{2}\left|y_{2}\right|^{p-2}\right\} \\
& \cdot\left\{\|x\|_{p}\|y\|_{p}-1-\lambda\left(\|x\|_{p}-1\right)\left(\|y\|_{p}-1\right)\right\} .
\end{aligned}
$$

It is obvious that

$$
\|x\|_{p}\|y\|_{p}-1-\lambda\left(\|x\|_{p}-1\right)\left(\|y\|_{p}-1\right) \geq 0
$$

for any $\lambda \in[0,1]$ and $\|x\|_{p},\|y\|_{p} \geq 1$. Thus, we have from Hölder's inequality that

$$
\begin{aligned}
& V(x, y)-V(T x, T y)-\lambda V(x-T x, y-T y) \\
& \geq\|x\|_{p}^{2}+\|y\|_{p}^{2}-2-\lambda\left(\|x\|_{p}-1\right)^{2}-\lambda\left(\|y\|_{p}-1\right)^{2} \\
&-2\|x\|_{p}^{-1}\|y\|_{p}^{1-p}\|x\|_{p}\|y\|_{p}^{p-1} \\
& \cdot\left\{\|x\|_{p}\|y\|_{p}-1-\lambda\left(\|x\|_{p}-1\right)\left(\|y\|_{p}-1\right)\right\} \\
&=\|x\|_{p}^{2}+\|y\|_{p}^{2}-2-\lambda\left(\|x\|_{p}-1\right)^{2}-\lambda\left(\|y\|_{p}-1\right)^{2} \\
&-2\left\{\|x\|_{p}\|y\|_{p}-1-\lambda\left(\|x\|_{p}-1\right)\left(\|y\|_{p}-1\right)\right\} \\
&=\|x\|_{p}^{2}+\|y\|_{p}^{2}-2-\lambda \\
& \cdot\left\{\|x\|_{p}^{2}-2\|x\|_{p}+1+\|y\|_{p}^{2}-2\|y\|_{p}+1\right\} \\
&-2\|x\|_{p}\|y\|_{p}+2+2 \lambda\left\{\|x\|_{p}\|y\|_{p}-\|x\|_{p}-\|y\|_{p}+1\right\} \\
&=\left(\|x\|_{p}-\|y\|_{p}\right)^{2}-\lambda\left(\|x\|_{p}-\|y\|_{p}\right)^{2} \\
&=(1-\lambda)\left(\|x\|_{p}-\|y\|_{p}\right)^{2} \geq 0, \quad \text { for any } \lambda \in[0,1] .
\end{aligned}
$$

That is, the inequality (1) holds.

It is clear that if $\|x\|_{p},\|y\|_{p} \leq 1$ then inequality (1) holds. Therefore, from Cases (a), (b), and (c), we obtain the conclusion that $T$ is $V$-strongly nonexpansive for any $\lambda \epsilon$ $(0,1]$. 
Remark 10. When $p=1$, we have given the result in [5]. When $p=2$, we already know that $E$ is a Hilbert space and a $V$-strongly nonexpansive mapping $T$ is nonexpansive.

Theorem 11. There exists a $V$-strongly nonexpansive mapping $T$ with a nonempty subset of fixed points such that $T$ is not nonexpansive for some Banach space.

Proof. It is enough to show that the $V$-strongly nonexpansive mapping which is given in the previous proposition is not nonexpansive.

Let $x=(0,1) \in E$. Suppose that $y=\left(y_{1}, y_{2}\right)$ satisfies that $\|y\|_{p}^{p}=\left|y_{1}\right|^{p}+\left|y_{2}\right|^{p}>1$ and $0<y_{1}, y_{2}<1$. Then $T y=\|y\|_{p}^{-1} y$. Let $h=\left(y_{2} / y_{1}\right)$ and $t=\|y\|_{p}^{-1} y_{1}-y_{1}$. We have that $t<0$ and $\|y\|_{p}^{-1} y_{2}-y_{2}=h t<0$. Then we obtain that $T y=\left(\|y\|_{p}^{-1} y_{1},\|y\|_{p}^{-1} h y_{1}\right)$. Then, we have that

$$
\begin{aligned}
\|T x-T y\|_{p}^{p} & =\left\|\left(-\|y\|_{p}^{-1} y_{1}, 1-\|y\|_{p}^{-1} h y_{1}\right)\right\|^{p} \\
& =\left|-\|y\|_{p}^{-1} y_{1}\right|^{p}+\left|1-\|y\|_{p}^{-1} h y_{1}\right|^{p} \\
& =\left(\|y\|_{p}^{-1} y_{1}\right)^{p}+\left(1-\|y\|_{p}^{-1} h y_{1}\right)^{p} \\
& =\left(y_{1}+t\right)^{p}+\left(1-h\left(y_{1}+t\right)\right)^{p},
\end{aligned}
$$

and since $\|x-y\|_{p}^{p}=y_{1}^{p}+\left(1-h y_{1}\right)^{p}$, we have that

$$
\begin{aligned}
& \|T x-T y\|_{p}^{p}-\|x-y\|_{p}^{p} \\
& =\left(y_{1}+t\right)^{p}-y_{1}^{p}+\left(1-h\left(y_{1}+t\right)\right)^{p}-\left(1-h y_{1}\right)^{p} .
\end{aligned}
$$

Therefore, we will show that

$$
\begin{aligned}
\|T x-T y\|_{p}^{p}-\|x-y\|_{p}^{p}>0 \\
\Longleftrightarrow\left(y_{1}+t\right)^{p}-y_{1}^{p}+\left(1-h\left(y_{1}+t\right)\right)^{p}-\left(1-h y_{1}\right)^{p}>0 \\
\Longleftrightarrow\left\{\left(y_{1}+t\right)^{p}-y_{1}^{p}\right\} t^{-1} \\
\quad+\left\{\left(1-h\left(y_{1}+t\right)\right)^{p}-\left(1-h y_{1}\right)^{p}\right\} t^{-1}<0,
\end{aligned}
$$

since $t<0$. Let $h$ be fixed. As $\|y\|_{p}^{p}=y_{1}^{p}+\left(h y_{1}\right)^{p} \rightarrow 1$, $t=\|y\|_{p}^{-1} y_{1}-y_{1} \rightarrow 0$. Thus, we have for a sufficiently small $|t|$ that

$$
\begin{aligned}
& \left\{\left(y_{1}+t\right)^{p}-y_{1}^{p}\right\} t^{-1} \\
& +\left\{\left(1-h\left(y_{1}+t\right)\right)^{p}-\left(1-h y_{1}\right)^{p}\right\} t^{-1}<0 \\
\Longleftrightarrow & p y_{1}^{p-1}-p h\left(1-h y_{1}\right)^{p-1}<0 .
\end{aligned}
$$

It is trivial that

$$
\begin{aligned}
p y_{1}^{p-1}-p h\left(1-h y_{1}\right)^{p-1}<0 & \Longleftrightarrow y_{1}^{p-1}<h\left(1-h y_{1}\right)^{p-1} \\
& \Longleftrightarrow y_{1}^{p}<y_{2}\left(1-y_{2}\right)^{p-1} .
\end{aligned}
$$

Let $p=3 / 2$. For $y=(0.2,0.95)$, we have that

$$
y_{1}^{p}=(0.2)^{3 / 2}<0.95(0.05)^{1 / 2}=y_{2}\left(1-y_{2}\right)^{p-1} .
$$

We obtain that $\|y\|_{p}^{p}=(0.2)^{3 / 2}+(0.95)^{3 / 2}>1$ and that

$$
\begin{aligned}
\|T x-T y\|_{p}^{p} & =\|y\|_{p}^{-p}\left\{(0.2)^{3 / 2}+\left(\|y\|_{p}-0.95\right)^{3 / 2}\right\} \\
& >(0.2)^{3 / 2}+(0.05)^{3 / 2}=\|x-y\|_{p}^{p} .
\end{aligned}
$$

Therefore, we obtain the conclusion.

We remark that the symbols $x_{n} \rightarrow u$ and $x_{n} \rightarrow u$ mean that $\left\{x_{n}\right\}$ converges strongly and weakly to $u$, respectively. We will introduce the following important lemmas for proofs of our theorems.

Lemma 12. (a) For all $x, y, z \in E$,

$$
\begin{aligned}
V(x, y) & \leq V(x, y)+V(y, z) \\
& =V(x, z)-2\langle x-y, J y-J z\rangle .
\end{aligned}
$$

(b) Let $\left\{x_{n}\right\}$ be a sequence in $E$ such that there exists $\lim _{n \rightarrow \infty} V\left(x_{n}, p\right)<\infty$ for some $p \in E$; then $\left\{x_{n}\right\}$ is bounded.

Lemma 13 (see [3]). Let $E$ be a smooth and uniformly convex Banach space and $C$ a nonempty, convex, and closed subset of E. Suppose that $T: C \rightarrow$ E satisfies

$$
V(T x, T y) \leq V(x, y) \quad \forall x, y \in C .
$$

If a weakly convergent sequence $\left\{z_{n}\right\}_{n \geq 1} \subset C$ satisfies that $\lim _{n \rightarrow \infty} V\left(T z_{n}, z_{n}\right)=0$, it holds that $z_{n} \rightarrow z \in F(T)$.

Theorem 14 (see $[1,11]$ ). Let $Y$ be a compact subset of $a$ topological vector space $E$ and let $X$ be a convex subset of $Y$. Let $A: X \rightarrow 2^{Y}$ be an operator such that, for each $y \in Y, A^{-1} y$ is convex. Suppose that $B: X \rightarrow 2^{Y}$ satisfies the following:

(1) $B x \subset A x$ for each $x \in X$,

(2) $B^{-1} y \neq \emptyset$ for each $y \in Y$,

(3) $B x$ is open for each $x \in X$.

Then there exists a point $x_{0} \in X$ such that $x_{0} \in A x_{0}$.

Lemma 15 (see [12]). Let $s>0$ and let $E$ be a Banach space. Then $E$ is uniformly convex if and only if there exists a continuous, strictly increasing, and convex function $g$ : $[0, \infty) \rightarrow[0, \infty), g(0)=0$, such that

$$
\|x+y\|^{2} \geq\|x\|^{2}+2\langle y, j\rangle+g(\|y\|)
$$

for all $x, y \in\{z \in E:\|z\| \leq s\}$ and $j \in J x$.

Lemma 16 (see [13]). Let $E$ be a smooth and uniformly convex Banach space. Then, there exists a continuous, strictly increasing, and convex function $g:[0, \infty) \rightarrow[0, \infty)$ such that $g(0)=0$ and, for each real number $r>0$,

$$
0 \leq g(\|x-y\|) \leq V(x, y)
$$

for all $x, y \in B_{r}=\{z \in E:\|z\| \leq r\}$. 
Lemma 17 (see [13]). Let E be a smooth and uniformly convex Banach space and $\left\{y_{n}\right\}$ and $\left\{z_{n}\right\}$ in $E$. If $\lim _{n \rightarrow \infty} V\left(y_{n}, z_{n}\right)=0$ and either $\left\{y_{n}\right\}$ or $\left\{z_{n}\right\}$ is bounded, then $\left\{y_{n}-z_{n}\right\} \rightarrow 0$.

\section{Main Results}

In this section, we prove a weak convergence theorem and strong convergence theorems for finding fixed points of a $V$ strongly nonexpansive mapping $T$ in Banach spaces, and then we show the existence theorem for fixed points of $T$ with a dissipative property (cf. [10]).

Theorem 18. Let $E$ be a smooth and uniformly convex Banach space and $C$ a nonempty, closed, and convex subset of $E$. Suppose that a mapping $T: C \rightarrow C$ is $V$-strongly nonexpansive with $\lambda$ and that $F(T) \neq \emptyset$. One defines a Mann iterative sequence $\left\{x_{n}\right\}$ as follows: for any $x_{1} \in C$ and $n \geq 1$,

$$
x_{n+1}=\beta_{n} x_{n}+\left(1-\beta_{n}\right) T x_{n},
$$

where $\left\{\beta_{n}\right\} \subset(0,1)$ and $\lim _{n \rightarrow \infty} \beta_{n}=0$. Then $x_{n} \rightarrow p_{0}$ for some $p_{0} \in F(T)$.

Proof. Suppose that $p \in F(T)$. Then we have from the convexity of $V$ that

$$
\begin{aligned}
V\left(x_{n+1}, p\right) & =V\left(\beta_{n} x_{n}+\left(1-\beta_{n}\right) T x_{n}, p\right) \\
& \leq \beta_{n} V\left(x_{n}, p\right)+\left(1-\beta_{n}\right) V\left(T x_{n}, p\right) \\
& =\beta_{n} V\left(x_{n}, p\right)+\left(1-\beta_{n}\right) V\left(T x_{n}, T p\right) .
\end{aligned}
$$

Since $T$ is $V$-strongly nonexpansive with $\lambda$, we have that

$$
\begin{aligned}
& V\left(x_{n+1}, p\right) \\
& \leq \beta_{n} V\left(x_{n}, p\right)+\left(1-\beta_{n}\right) \\
& \cdot\left\{V\left(x_{n}, p\right)-\lambda V\left((I-T) x_{n},(I-T) p\right)\right\} \\
&= V\left(x_{n}, p\right)-\left(1-\beta_{n}\right) \lambda V\left(x_{n}-T x_{n}, 0\right) \\
& \leq V\left(x_{n}, p\right) .
\end{aligned}
$$

Hence, we have $\lim _{n \rightarrow \infty} V\left(x_{n}, p\right)=\alpha<\infty$. From Lemma 12 (b), $\left\{x_{n}\right\}$ is bounded. Furthermore, we have that

$$
\left(1-\beta_{n}\right) \lambda V\left(x_{n}-T x_{n}, 0\right) \leq V\left(x_{n}, p\right)-V\left(x_{n+1}, p\right) .
$$

Since $\lim _{n \rightarrow \infty} \beta_{n}=\lim _{n \rightarrow \infty}\left\{V\left(x_{n}, p\right)-V\left(x_{n+1}, p\right)\right\}=0$, we obtain that

$$
\lim _{n \rightarrow \infty} V\left(x_{n}-T x_{n}, 0\right)=\lim _{n \rightarrow \infty}\left\|x_{n}-T x_{n}\right\|^{2}=0 .
$$

This means that $\left\{x_{n}-T x_{n}\right\}$ converges strongly to 0 . Hence, $\left\{T x_{n}\right\}$ is also bounded, and there exists $M>0$ such that $\left\|x_{n}\right\|,\left\|T x_{n}\right\| \leq M-\|p\|$ for all $n \geq 1$.
On the other hand, we have from Lemma 12 (a) that

$$
\begin{aligned}
0 \leq & V\left(x_{n}, T x_{n}\right) \\
= & V\left(x_{n}, p\right)-V\left(T x_{n}, p\right)-2\left\langle x_{n}-T x_{n}, J T x_{n}-J p\right\rangle \\
\leq & V\left(x_{n}, p\right)-V\left(T x_{n}, p\right)+2\left\|x_{n}-T x_{n}\right\|\left(\left\|T x_{n}\right\|+\|p\|\right) \\
\leq & V\left(x_{n}, p\right)-V\left(T x_{n}, p\right)+2 M\left\|x_{n}-T x_{n}\right\| \\
= & \left\|x_{n}\right\|^{2}-\left\|T x_{n}\right\|^{2}-2\left\langle x_{n}-T x_{n}, J p\right\rangle+2 M\left\|x_{n}-T x_{n}\right\| \\
= & \left(\left\|x_{n}\right\|-\left\|T x_{n}\right\|\right)\left(\left\|x_{n}\right\|+\left\|T x_{n}\right\|\right) \\
& -2\left\langle x_{n}-T x_{n}, J p\right\rangle+2 M\left\|x_{n}-T x_{n}\right\| \\
\leq & \left\|x_{n}-T x_{n}\right\|\left(\left\|x_{n}\right\|+\left\|T x_{n}\right\|+2 M\right)-2\left\langle x_{n}-T x_{n}, J p\right\rangle .
\end{aligned}
$$

Hence, we obtain that $\lim _{n \rightarrow \infty} V\left(x_{n}, T x_{n}\right)=\lim _{n \rightarrow \infty} V\left(T x_{n}\right.$, $\left.x_{n}\right)=0$. From Lemma 13, there exists a point $p_{0} \in F(T)$ such that $x_{n} \rightarrow p_{0}$ and $T x_{n} \rightarrow p_{0}$.

The duality mapping $J$ of a Banach space $E$ with Gâteaux differentiable norm is said to be weakly sequentially continuous if $x_{n} \rightarrow x$ in $E$ implies that $\left\{J x_{n}\right\}$ converges weak star to $J x$ in $E^{*}$ (cf. [14]). This happens, for example, if $E$ is a Hilbert space, or finite-dimensional and smooth, or $l^{p}$ if $1<p<\infty$ (cf. [15]). Next we prove a strong convergence theorem.

Theorem 19. Let $E$ be a reflexive, smooth, and strictly convex Banach space. Suppose that the duality mapping J of E is weakly sequentially continuous. Suppose that $C$ is a nonempty, closed, and convex subset of $E, T: C \rightarrow C$ is $V$-strongly nonexpansive with $\lambda$, and $F(T) \neq \emptyset$. One defines a Mann iterative sequence $\left\{x_{n}\right\}$ as follows: for any $x_{1} \in C$ and $n \geq 1$,

$$
x_{n+1}=\beta_{n} x_{n}+\left(1-\beta_{n}\right) T x_{n},
$$

where $\left\{\beta_{n}\right\} \subset(0,1)$ and $\lim _{n \rightarrow \infty} \beta_{n}=0$. If $T$ satisfies that

$$
\langle x, J T x\rangle \leq 0 \quad \forall x \in C,
$$

then $x_{n} \rightarrow p_{0}$ and $T x_{n} \rightarrow p_{0}$ for some $p_{0} \in F(T)$.

Proof. As in the proof of Theorem 18, we obtain that $\lim _{n \rightarrow \infty} V\left(x_{n}, T x_{n}\right)=0$ and $x_{n} \rightarrow p_{0}$ and $T x_{n} \rightarrow p_{0}$ for some $p_{0} \in F(T)$. Furthermore, from Lemma 12 (a), we have that

$$
\begin{aligned}
0 \leq & V\left(x_{n}, p_{0}\right)+V\left(p_{0}, T x_{n}\right) \\
= & V\left(x_{n}, T x_{n}\right)-2\left\langle x_{n}-p_{0}, J p_{0}-J T x_{n}\right\rangle \\
= & V\left(x_{n}, T x_{n}\right)-2\left\langle x_{n}-p_{0}, J p_{0}\right\rangle \\
& +2\left\langle x_{n}, J T x_{n}\right\rangle-2\left\langle p_{0}, J T x_{n}\right\rangle .
\end{aligned}
$$

Hence, the assumptions imply that

$$
V\left(x_{n}, p_{0}\right) \longrightarrow 0, \quad V\left(p_{0}, T x_{n}\right) \longrightarrow 0 \quad \text { as } n \longrightarrow \infty .
$$

From Lemma 17, we have the conclusion that $x_{n} \rightarrow p_{0}$ and $T x_{n} \rightarrow p_{0}$. 
Condition (44) is a definition of a linear dissipative mapping $T$ (cf. [16]). Moreover, we give a definition of a $J$-dissipative mapping for nonlinear mappings in a Banach space.

Definition 20. Let $J$ be a single-valued duality mapping on $E$ and let $C$ be a nonempty subset of $E$. Then a mapping $T$ : $C \rightarrow E$ is called $J$-dissipative if it holds that

$$
\langle x-y, J T x-J T y\rangle \leq 0
$$

for all $x, y \in C$.

In a Hilbert space, such a mapping $T$ is called dissipative. In Banach spaces, we remark that the $J$-dissipative mapping is not equal to the dissipative mapping (cf. [17]). Next we give a characterization of $J$-dissipative mappings by using $V(\cdot, \cdot)$.

Theorem 21. Let $E$ be a smooth Banach space, $C$ a nonempty subset of $E$, and $T: C \rightarrow E$ a mapping. Then, the following are equivalent.

(a) $T$ is J-dissipative.

(b) For all $x, y \in C$,

$$
V(x, T y)+V(y, T x) \leq V(x, T x)+V(y, T y) .
$$

Proof. For any $x, y \in C$,

$$
\langle x-y, J T x-J T y\rangle \leq 0
$$

is equal to

$$
\begin{aligned}
& -2\langle x, J T y\rangle-2\langle y, J T x\rangle \leq-2\langle x, J T x\rangle-2\langle y, J T y\rangle, \\
& -2\langle x, J T y\rangle-2\langle y, J T x\rangle+\|x\|^{2}+\|T y\|^{2}+\|y\|^{2}+\|T x\|^{2} \\
& \leq-2\langle x, J T x\rangle-2\langle y, J T y\rangle+\|x\|^{2}+\|T x\|^{2} \\
& +\|y\|^{2}+\|T y\|^{2} .
\end{aligned}
$$

From the definition of $V$, this inequality is equivalent to

$$
V(x, T y)+V(y, T x) \leq V(x, T x)+V(y, T y) .
$$
rem.

Furthermore, we have the following result by this theo-

Lemma 22. Suppose that $E$ is a smooth and strictly convex Banach space and that $C \subset E$ is a nonempty convex subset. Assume that a mapping $T: C \rightarrow E$ is J-dissipative. If there are fixed points of $T$, then $F(T)$ is singleton.

Proof. Assume that there exist $p_{0}$ and $q_{0}$ such that $T p_{0}=p_{0}$ and $T q_{0}=q_{0}$. Since $T$ is $J$-dissipative, we have by Theorem 21 that

$$
\begin{aligned}
0 & \leq V\left(p_{0}, T q_{0}\right)+V\left(q_{0}, T p_{0}\right) \\
& \leq V\left(p_{0}, T p_{0}\right)+V\left(q_{0}, T q_{0}\right) \\
& =V\left(p_{0}, p_{0}\right)+V\left(q_{0}, q_{0}\right)=0 .
\end{aligned}
$$

Thus, we have that $V\left(p_{0}, q_{0}\right)=V\left(q_{0}, p_{0}\right)=0$. This implies that

$$
\begin{gathered}
0 \leq\left(\left\|p_{0}\right\|-\left\|q_{0}\right\|\right)^{2} \leq V\left(p_{0}, q_{0}\right)=0, \\
\left\|p_{0}\right\|=\left\|q_{0}\right\| .
\end{gathered}
$$

Furthermore, we have

$$
\begin{aligned}
V\left(p_{0}, q_{0}\right) & =\left\|p_{0}\right\|^{2}+\left\|q_{0}\right\|^{2}-2\left\langle p_{0}, J q_{0}\right\rangle \\
& =\left\|p_{0}\right\|^{2}+\left\|p_{0}\right\|^{2}-2\left\langle p_{0}, J q_{0}\right\rangle=0,
\end{aligned}
$$

and we have $\left\|p_{0}\right\|^{2}=\left\langle p_{0}, J q_{0}\right\rangle$. Since $E$ is strictly convex and $J$ is one-to-one, we obtain that $p_{0}=q_{0}$.

We give a result before proving an existence theorem for fixed points.

Theorem 23 (see [10]). Let $E$ be a smooth and uniformly convex Banach space, and let $T: E \rightarrow E$ be a $V$-strongly nonexpansive mapping with $\lambda$. Then, one has that

$$
\lim _{\|x-y\| \rightarrow 0}\|T x-T y\|=0,
$$

for $\|x\|,\|y\|,\|T x\|,\|T y\| \leq r$, where $r>0$.

Proof. Since $T$ is a $V$-strongly nonexpansive with $\lambda$, we have

$$
\begin{aligned}
0 & \leq V(T x, T y)+\lambda V(x-T x, y-T y) \\
& \leq V(x, y) \\
& =\|x\|^{2}+\|y\|^{2}-2\langle x, J y\rangle \\
& =\|x\|^{2}-\|y\|^{2}-2\langle x-y, J y\rangle \\
& \leq\|x-y\|(\|x\|+\|y\|+2\|y\|) \\
& =\|x-y\|(\|x\|+3\|y\|), \quad \text { for any } x, y \in E .
\end{aligned}
$$

Thus, we obtain, for $x, y$ with $\|x\|,\|y\| \leq r$,

$$
\begin{gathered}
V(T x, T y) \longrightarrow 0, \\
V(x-T x, y-T y) \longrightarrow 0 \quad \text { as }\|x-y\| \longrightarrow 0 .
\end{gathered}
$$

From Lemma 16, we have that

$$
0 \leq g(\|T x-T y\|) \leq V(T x, T y) .
$$

Therefore, we have from (57) that $\lim _{\|x-y\| \rightarrow 0} g(\|T x-T y\|)=$ 0 . From the definition of $g$, we obtain that

$$
\lim _{\|x-y\| \rightarrow 0}\|T x-T y\|=0 .
$$

Remark 24. If $x \in E$ satisfies that $\|T x\|<r_{0}$ for $r_{0}>0$, the (57) implies that $\|T y\|<r_{0}+1$ for $y$ in the neighborhood of $x$. 
We will prove the following existence theorem by using Theorem 14.

Theorem 25. Let $E$ be a reflexive, strictly convex, and smooth Banach space and $C$ a nonempty, bounded, closed, and convex subset of E. Suppose $T: C \rightarrow C$ is a $V$-strongly nonexpansive and J-dissipative mapping. Then, there exists a unique fixed point of $T$.

Proof. At first, we will show that there exists $y_{0} \in C$ such that

$$
\left\{x \in C: V(x, T x)<V\left(y_{0}, T x\right)\right\}=\emptyset .
$$

Assume that, for all $y \in C$,

$$
\{x \in C: V(x, T x)<V(y, T x)\} \neq \emptyset .
$$

Let $A x=\{y \in C: V(x, T y)<V(y, T y)\}$ and $B x=\{y \in$ $C: V(x, T x)<V(y, T x)\}$ for all $x \in C$. Then, from the assumption, $B^{-1} y$ is nonempty for all $y \in C$. Since $T$ is $J$ dissipative, Theorem 21 implies that

$$
V(x, T y)-V(y, T y) \leq V(x, T x)-V(y, T x)
$$

for all $y \in B x$. This means that $B x \subset A x$ for any $x \in C$. For any $y \in C$, let $v_{j} \in A^{-1} y$ with $j \in\{1,2, \ldots, n\}$, and suppose that $v=\sum_{j=1}^{n} \alpha_{j} v_{j}$ and $\sum_{j=1}^{n} \alpha_{j}=1$ with $\alpha_{j}>0$. From the convexity of $V$, we have

$$
\begin{aligned}
V(v, T y) & =V\left(\sum_{j=1}^{n} \alpha_{j} v_{j}, T y\right) \leq \sum_{j=1}^{n} \alpha_{j} V\left(v_{j}, T y\right) \\
& \leq \sum_{j=1}^{n} \alpha_{j} V(y, T y)=V(y, T y) .
\end{aligned}
$$

Thus, we obtain that $A^{-1} y$ is convex for all $y \in C$. Since it is obvious that $B x$ is open for each $x \in C$, Theorem 14 implies that there exists a point $x_{0} \in C$ such that $x_{0} \in A x_{0}$. This means that

$$
V\left(x_{0}, T x_{0}\right)<V\left(x_{0}, T x_{0}\right)
$$

This is a contradiction. Thus, we have for some $y_{0} \in C$ that

$$
\left\{x \in C: V(x, T x)<V\left(y_{0}, T x\right)\right\}=\emptyset .
$$

This means that there exists $y_{0} \in C$ such that

$$
V\left(y_{0}, T x\right) \leq V(x, T x)
$$

for all $x \in C$.

Furthermore, we will show $V\left(y_{0}, T y_{0}\right) \leq V\left(x, T y_{0}\right)$ for all $x \in C$ if $y_{0}$ satisfies $(66)$. Let $y_{t}=(1-t) y_{0}+t x$ for any $t \in(0,1)$ and $x \in C$. Since $C$ is convex, then $y_{t} \in C$. Thus, we obtain that

$$
\begin{aligned}
V\left(y_{0}, T y_{t}\right) & \leq V\left(y_{t}, T y_{t}\right) \\
& =V\left((1-t) y_{0}+t x, T y_{t}\right)
\end{aligned}
$$

From the convexity of $V(\cdot, y)$ for $y \in C$,

$$
V\left(y_{0}, T y_{t}\right) \leq(1-t) V\left(y_{0}, T y_{t}\right)+t V\left(x, T y_{t}\right)
$$

and we have $V\left(y_{0}, T y_{t}\right) \leq V\left(x, T y_{t}\right)$. From the definition of $V(\cdot, \cdot)$, we have that

$$
\begin{aligned}
& \left|V\left(x, T y_{t}\right)-V\left(x, T y_{0}\right)\right| \\
& \quad=\left|\left\|T y_{t}\right\|^{2}-\left\|T y_{0}\right\|^{2}-2\left\langle x, J T y_{t}-J T y_{0}\right\rangle\right| \\
& \quad \leq\left(\left\|T y_{t}\right\|+\left\|T y_{0}\right\|\right)\left\|T y_{t}-T y_{0}\right\|+2\|x\|\left\|J T y_{t}-J T y_{0}\right\| .
\end{aligned}
$$

Therefore, we have, by Theorem 23 and the continuity of $J$ on a smooth Banach space, that $\lim _{t \rightarrow 0+} V\left(x, T y_{t}\right)=V\left(x, T y_{0}\right)$ and

$$
\begin{aligned}
V\left(y_{0}, T y_{0}\right) & =\lim _{t \rightarrow 0+} V\left(y_{0}, T y_{t}\right) \\
& \leq \lim _{t \rightarrow 0+} V\left(x, T y_{t}\right)=V\left(x, T y_{0}\right)
\end{aligned}
$$

for all $x \in C$. Letting $x=T y_{0}$, we have that

$$
V\left(y_{0}, T y_{0}\right) \leq V\left(T y_{0}, T y_{0}\right)=0
$$

Hence, $V\left(y_{0}, T y_{0}\right)=0$. This implies that

$$
\left\|y_{0}\right\|^{2}+\left\|T y_{0}\right\|^{2}=2\left\langle y_{0}, J T y_{0}\right\rangle \leq 2\left\|y_{0}\right\|\left\|T y_{0}\right\|,
$$

and then we obtain that

$$
\left(\left\|y_{0}\right\|-\left\|T y_{0}\right\|\right)^{2} \leq 0
$$

Thus, we have $\left\|y_{0}\right\|=\left\|T y_{0}\right\|$ and we have by (72) that $\left\|y_{0}\right\|^{2}=$ $\left\langle y_{0}, J T y_{0}\right\rangle$. Since $J$ is one-to-one on a strictly convex Banach space, $J T y_{0}=J y_{0}$ implies that $T y_{0}=y_{0}$. Therefore, we have the conclusion.

Finally, we will prove a strong convergence theorem for finding fixed points of a $V$-strongly nonexpansive mapping $T$ in a Banach space, without the assumption that $F(T) \neq \emptyset$.

Theorem 26. Let $E$ be a smooth and uniformly convex Banach space, and let $C$ be a nonempty, compact, and convex subset of $E$. Suppose that $T: C \rightarrow C$ is J-dissipative and $V$-strongly nonexpansive with $\lambda$. One defines a Mann iterative sequence $\left\{x_{n}\right\}$ as follows: for any $x_{1} \in C$ and $n \geq 1$,

$$
x_{n+1}=\beta_{n} x_{n}+\left(1-\beta_{n}\right) T x_{n}
$$

where $\left\{\beta_{n}\right\} \subset(0,1)$ and $\lim _{n \rightarrow \infty} \beta_{n}=0$. Then, there exists a unique fixed point $p_{0} \in C$ such that $x_{n} \rightarrow p_{0}$ and T $x_{n} \rightarrow p_{0}$.

Proof. From Theorem 25, we have that $F(T) \neq \emptyset$. As in the proof of Theorem 18, we obtain that $\lim _{n \rightarrow \infty} V\left(x_{n}, T x_{n}\right)=0$ and that there exists a point $p_{0} \in F(T)$ such that $x_{n} \rightarrow p_{0}$ and $T x_{n} \rightarrow p_{0}$. Since $T$ is $J$-dissipative, Theorem 21 implies that

$$
0 \leq V\left(x_{n}, T p_{0}\right)+V\left(p_{0}, T x_{n}\right) \leq V\left(x_{n}, T x_{n}\right)+V\left(p_{0}, T p_{0}\right) .
$$


From $T p_{0}=p_{0}$, we have for $n \geq 1$ that

$$
\begin{aligned}
0 & \leq V\left(x_{n}, p_{0}\right)+V\left(p_{0}, T x_{n}\right) \\
& \leq V\left(x_{n}, T x_{n}\right)+V\left(p_{0}, p_{0}\right)=V\left(x_{n}, T x_{n}\right) .
\end{aligned}
$$

Since $\lim _{n \rightarrow \infty} V\left(x_{n}, T x_{n}\right)=0$, we have that

$$
\lim _{n \rightarrow \infty} V\left(x_{n}, p_{0}\right)=\lim _{n \rightarrow \infty} V\left(p_{0}, T x_{n}\right)=0 .
$$

By Lemma 17, we obtain that $x_{n} \rightarrow p_{0}$ and $T x_{n} \rightarrow p_{0}$. We have the conclusion.

\section{Conflict of Interests}

The author declares that there is no conflict of interests regarding the publication of this paper.

\section{Acknowledgments}

The author would like to thank the reviewers of this paper for their enduring reading and Professor N. Hirano and Professor A. T. Lau for their encouragement. Finally, the author deeply appreciates her mother Ayako's hearty support.

\section{References}

[1] W. Takahashi, Nonlinear Functional Analysis, Fixed Point Theory and its Applications, Yokohama Publishers, Yokohama, Japan, 2000.

[2] Y. I. Alber and S. Guerre-Delabriere, "Principle of weakly contractive maps in Hilbert spaces," in New Results in Operator Theory and Its Applications, vol. 98 of Operator Theory: Advances and Applications, pp. 7-22, Birkhäuser, Basel, Switzerland, 1997.

[3] D. Butnariu and E. Resmerita, "Bregman distances, totally convex functions, and a method for solving operator equations in Banach spaces," Abstract and Applied Analysis, vol. 2006, Article ID 84919, 39 pages, 2006.

[4] T. Ibaraki and W. Takahashi, "A new projection and convergence theorems for the projections in Banach spaces," Journal of Approximation Theory, vol. 149, no. 1, pp. 1-14, 2007.

[5] H. Manaka, "Convergence theorems for a maximal monotone operator and a $V$-strongly nonexpansive mapping in a Banach space," Abstract and Applied Analysis, vol. 2010, Article ID 189814, 20 pages, 2010.

[6] S. Reich, "Iterative methods for accretive sets," in Nonlinear Equations in Abstract Spaces, pp. 317-326, Academic Press, New York, NY, USA, 1978.

[7] H. H. Bauschke, "Fenchel duality, Fitzpatrick functions and the extension of firmly nonexpansive mappings," Proceedings of the American Mathematical Society, vol. 135, no. 1, pp. 135-139, 2007.

[8] R. E. Bruck and S. Reich, "Nonexpansive projections and resolvents of accretive operators in Banach spaces," Houston Journal of Mathematics, vol. 3, no. 4, pp. 459-470, 1977.

[9] K. Goebel and W. A. Kirk, Topics in Metric Fixed Point Theory, vol. 28 of Cambridge Studies in Advanced Mathematics, Cambridge University Press, Cambridge, UK, 1990.

[10] H. Manaka, Convergence theorems for fixed points with iterative methods in Banach spaces [Thesis], Yokohama National University, 2011.
[11] W. Takahashi, "Fixed point, minimax, and Hahn-Banach theorems," Proceedings of Symposia in Pure Mathematics, vol. 45, part 2, pp. 419-427, 1986.

[12] H. K. Xu, "Inequalities in Banach spaces with applications," Nonlinear Analysis: Theory, Methods \& Applications, vol. 16, no. 12, pp. 1127-1138, 1991.

[13] S. Kamimura and W. Takahashi, "Strong convergence of a proximal-type algorithm in a Banach space," SIAM Journal on Optimization, vol. 13, no. 3, pp. 938-945, 2002.

[14] S. Reich, "Approximating zeros of accretive operators," Proceedings of the American Mathematical Society, vol. 51, no. 2, pp. 381384, 1975.

[15] F. E. Browder, "Fixed point theorems for nonlinear semicontractive mappings in Banach spaces," Archive for Rational Mechanics and Analysis, vol. 21, no. 4, pp. 259-269, 1966.

[16] H. O. Fattorini, Encyclopedia of Mathematics and its Applications, vol. 18 of Encyclopedia of Mathematics and its Applications, Addison-Wesley, New York, NY, USA, 1983.

[17] I. Miyadera, Hisenkei hangun (Kinokuniya Suugaku gansyo 10), Kinokuniya shoten, 1977 (Japanese). 


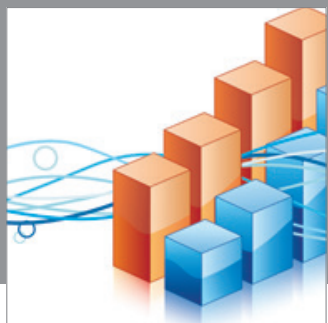

Advances in

Operations Research

mansans

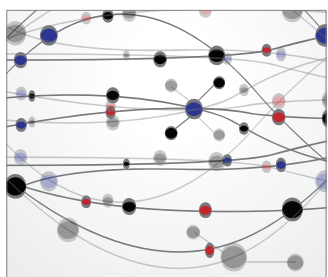

The Scientific World Journal
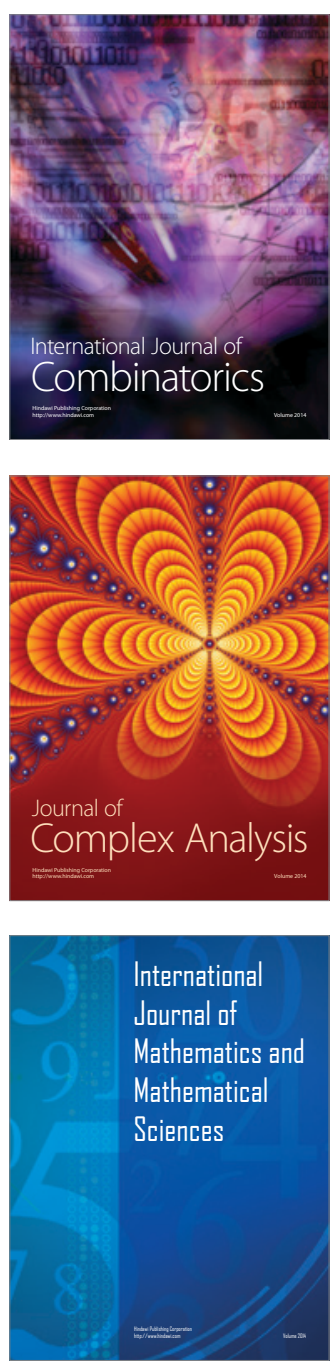
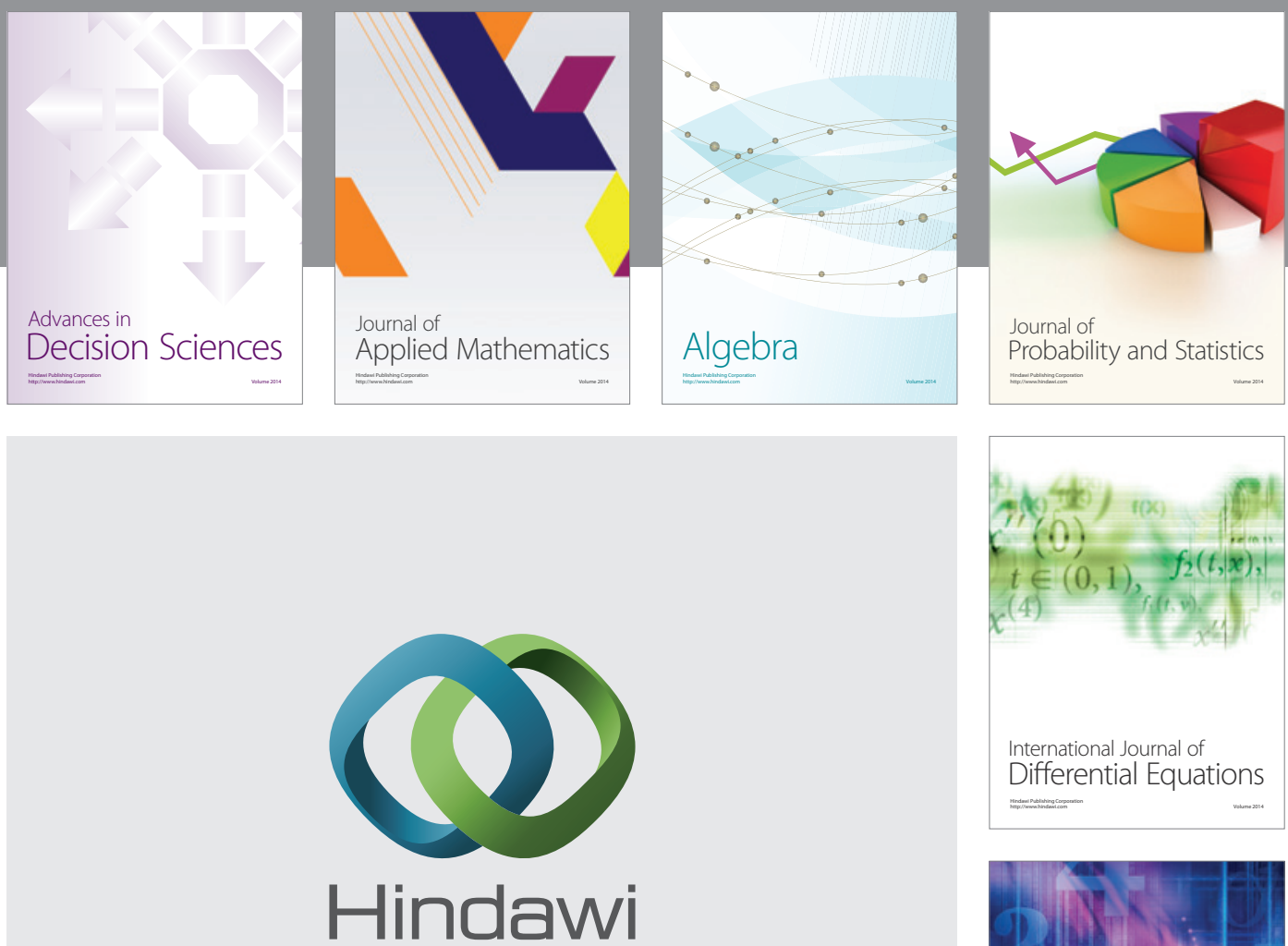

Submit your manuscripts at http://www.hindawi.com
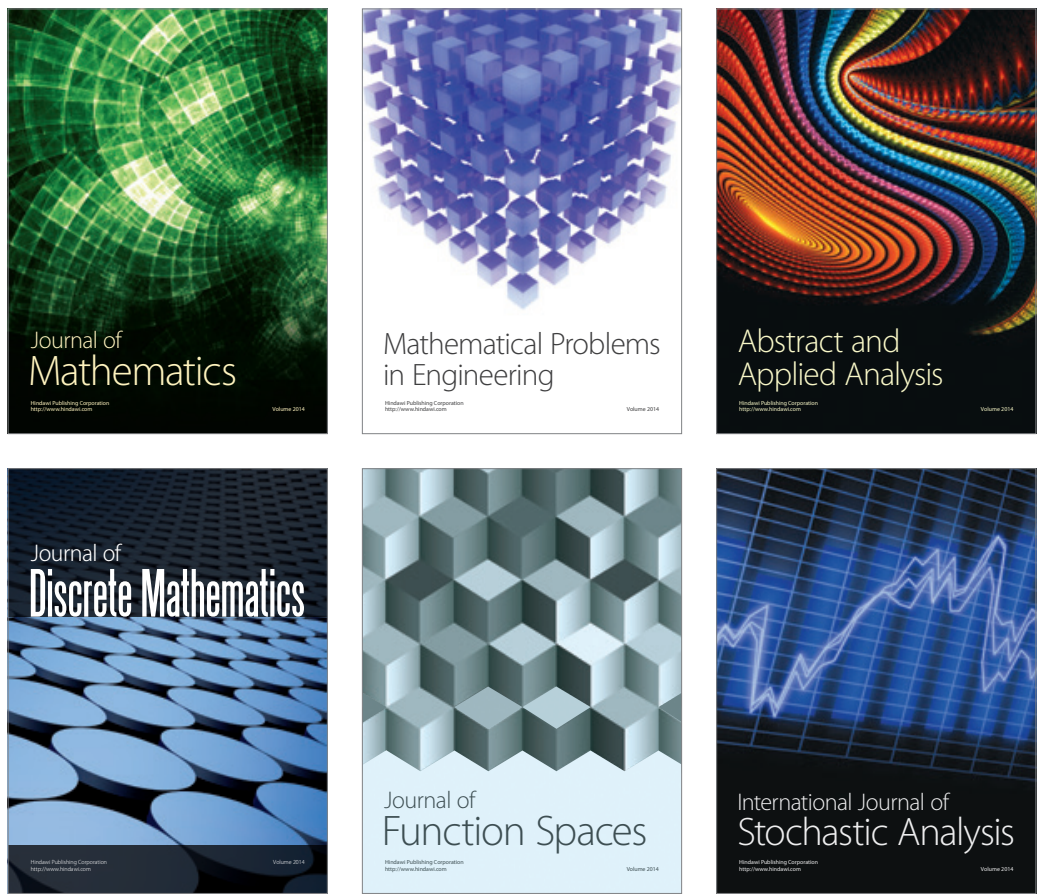

Journal of

Function Spaces

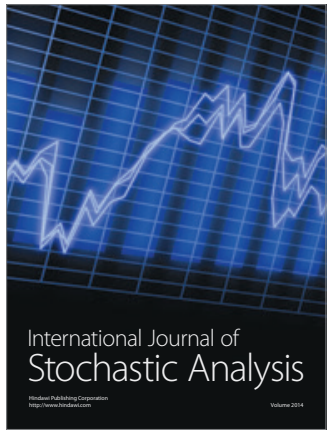

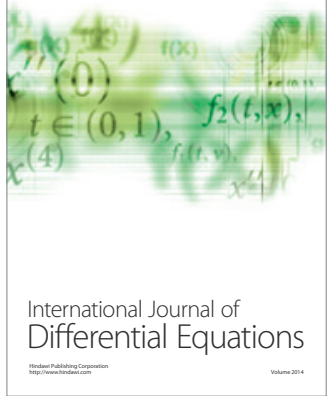
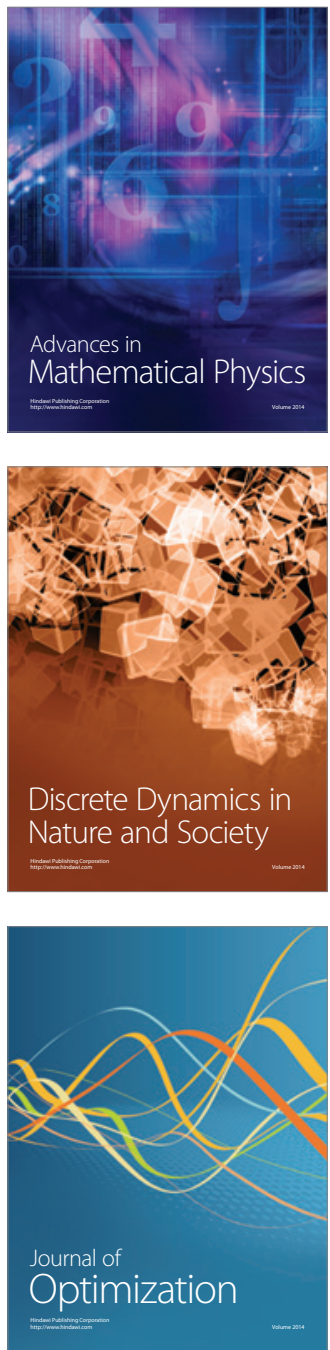